Relations industrielles

Industrial Relations

\title{
Les jeunes face aux conditions et au milieu de travail, Genève, Bureau international du travail, 1977, 40 pp.
}

\section{Jean-Pierre Auger}

Volume 33, numéro 3, 1978

URI : https://id.erudit.org/iderudit/028905ar

DOI : https://doi.org/10.7202/028905ar

Aller au sommaire du numéro

Éditeur(s)

Département des relations industrielles de l'Université Laval

ISSN

0034-379X (imprimé)

1703-8138 (numérique)

Découvrir la revue

Citer ce compte rendu

Auger, J.-P. (1978). Compte rendu de [Les jeunes face aux conditions et au milieu de travail, Genève, Bureau international du travail, 1977, 40 pp.] Relations industrielles / Industrial Relations, 33(3), 575-575.

https://doi.org/10.7202/028905ar

Tous droits réservés @ C Département des relations industrielles de l'Université Laval, 1978
Ce document est protégé par la loi sur le droit d'auteur. L'utilisation des services d'Érudit (y compris la reproduction) est assujettie à sa politique d'utilisation que vous pouvez consulter en ligne.

https://apropos.erudit.org/fr/usagers/politique-dutilisation/ 
mesures déjà prises dans plusieurs pays industrialisés et d'obtenir rapidement un état de la question. Pour approfondir le sujet et obtenir plus d'informations sur des expériences précises d'amélioration de la vie au travail, on consultera avec profit l'ouvrage de Louis E. Davis et Albert B. Cherns intitulé The Quality of Working Life, New York, The Free Press, 1975, 2 vol. En langue française, la revue Sociologie du travail a publié deux dossiers sur les questions reliées à l'organisation du travail (1974, no. 4 et 1976, no. 1); la revue Économie et Humanisme en a fait autant dans son numéro 227, juin-février 1976. Enfin, rappelons que le $32 \mathrm{e}$ Congrès annuel du département des relations industrielles de l'Université Laval, en 1977, fut consacré à l'un des aspects important de cette question, sous le thème «Participation et négociation collective».

Alain VINET

Université Laval

Les jeunes face aux conditions et au milieu de travail, Genève, Bureau international du travail, $1977,40 \mathrm{pp}$.

Le document du B.I.T. a été préparé en tant que document de travail pour le colloque sur «la qualité du travail et ses perspectives" tenu à Paris en juin 1976. Il s'agissait donc dans ce document de présenter les principaux problèmes des jeunes face à leur vie de travail. Pour ce faire, le texte a été divisé en trois chapitres: d'abord, une présentation des conditions actuelles de travail des jeunes; puis les attitudes, réactions et comportements des jeunes face à ces conditions de travail; et enfin des voies de solution pour une vie de travail mieux adaptée aux attentes de la jeunesse.

Règle générale, à peu près tout l'ensemble des conditions de travail ne permettent pas une insertion facile des jeunes dans la vie de travail. Dès l'embauche, où l'employeur est réticent à engager un jeune, jusqu'à la participation des jeunes travailleurs à la vie de l'entreprise (comité d'entreprise) ou à la vie syndicale, et en passant par tout ce qu'il y a entre les deux (organisation du temps de travail, cadences, nature des travaux, sécurité et hygiène professionnelles, rémunération, logement, alimentation), rien n'est pensé spécifiquement pour eux.

Le divorce entre ces conditions réelles de travail et les attentes des jeunes est énorme, ce qui provoque une marginalisation des jeunes travailleurs. Celle-ci entraîne à son tour des attitudes de rejet: instabilité, absentéisme, désintérêt au travail provoquent des pertes, refus de formation.

Face à cela, le document du B.I.T. propose, dans le troisième chapitre, des solutions d'ordre général et qui tombent sous le sens commun: améliorer le placement en vue de faciliter l'embauche des jeunes, atténuer la pénibilité des tâches qui leur sont confiées, diminuer le temps de travail, augmenter leur rémunération, améliorer l'hygiène et la sécurité professionnelles, favoriser leur participation à la vie de l'entreprise et à la vie syndicale.

Bien qu'il s'agisse d'un document de travail, donc sujet à discussions et à nuances, le document du B.I.T. pêche au moins à deux niveaux: l'identification des problèmes auxquels a à faire face la jeunesse et l'étude de son comportement visà-vis ces problèmes sont tellement globales qu'on a peine à croire qu'il s'agit là de la réalité. De même, les solutions avancées sont tellement peu opérationnelles qu'elles sont idéalistes et édémiques.

Il serait peut-être souhaitable qu'un organisme international comme le B.I.T. cesse de véhiculer de telles généralités et qu'il étudie plus en profondeur dans un seul pays au besoin, les problèmes du travail, et ceux des jeunes en particulier, très importants par ailleurs, il est impossible de le nier.

Jean Pierre AUGER

Université Laval

Small is Beautiful. Une société à la mesure de l'Homme, par E.F. Schumacher, Paris, Contretemps/Le Seuil, 1978, $316 \mathrm{pp}$.

Ce livre, d'une beauté et d'une fraîcheur exceptionnelles, offre, dans un langage 